\title{
Article
}

\section{Skipping of the very-high-frequency structural particle de $(\square)$ in Chinese reading}

Zang, Chuanli, Zhang, Manman, Bai, Xuejun, Yan, Guoli, Angele, Bernhard and Liversedge, Simon Paul

Available at http://clok.uclan.ac.uk/26465/

Zang, Chuanli ORCID: 0000-0002-9573-4968, Zhang, Manman, Bai, Xuejun, Yan, Guoli, Angele, Bernhard and Liversedge, Simon Paul ORCID: 0000-00028579-8546 (2018) Skipping of the very-high-frequency structural particle de $(\square)$ in Chinese reading. Quarterly Journal of Experimental Psychology, 71 (1). pp. 152-160. ISSN 1747-0218

It is advisable to refer to the publisher's version if you intend to cite from the work. $10.1080 \% 2 \mathrm{~F} 17470218.2016 .1272617$

For more information about UCLan's research in this area go to http://www.uclan.ac.uk/researchgroups/ and search for < name of research Group>.

For information about Research generally at UCLan please go to http://www.uclan.ac.uk/research/

All outputs in CLoK are protected by Intellectual Property Rights law, including Copyright law. Copyright, IPR and Moral Rights for the works on this site are retained by the individual authors and/or other copyright owners. Terms and conditions for use of this material are defined in the policies page.

\section{CLoK}

Central Lancashire online Knowledge www.clok.uclan.ac.uk

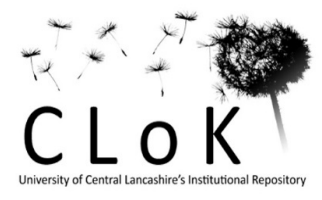




\title{
Skipping of the Very High Frequency Structural \\ Particle de (的) in Chinese Reading
}

\author{
Chuanli Zang ${ }^{1}$, Manman Zhang ${ }^{1}$, Xuejun Bai ${ }^{1}$, Guoli Yan ${ }^{1}$, \\ Bernhard Angele ${ }^{2}$, Simon P. Liversedge ${ }^{3}$ \\ ${ }^{1}$ Tianjin Normal University, P.R. China \\ ${ }^{2}$ Bournemouth University, UK \\ ${ }^{3}$ University of Southampton, UK
}

Send Correspondence to:

Simon P. Liversedge,

Centre for Visual Cognition,

School of Psychology, Shackleton Building,

University of Southampton,

Highfield, Southampton, SO17 1BJ, UK

Email: s.p.liversedge@soton.ac.uk

Phone: +44 2380599399 Fax: +44 2380594597

Running Head: Skipping of Chinese Structural Particle 


\begin{abstract}
English readers do not fixate every word: during their first pass through a sentence they skip a third of the words (Rayner, 1998, 2009). How do readers decide whether to skip or fixate a word? Angele and Rayner (2013) showed that English readers base skipping decisions on the parafoveal information available, but not the sentential context. Due to the increased visual density of the language, Chinese readers may be able to process a parafoveal word and integrate it with the sentence context to a greater extent than English readers. Consequently, influences on skipping decisions in Chinese may differ from those in English. In a boundary paradigm (Rayner, 1975) experiment, participants read sentences containing a single-character target verb (e.g., 取 meaning get) whose preview was manipulated in three conditions: identity preview; a preview consisting of the syntactically anomalous high frequency structural particle de (的), or a pseudocharacter preview. The results showed that Chinese readers were more likely to skip the target when the preview was de than in either of the other conditions, suggesting that de-skipping is triggered by the parafoveal preview of a highly frequent particle word rather than on the likelihood of the upcoming word given the sentential context. The present study shows that this phenomenon is observable across languages and writing systems with different degrees of visual density.
\end{abstract}

Keywords: Eye movements, skipping, structural particle, Chinese reading. 
It is well documented that not all words in a sentence are fixated, and a word is said to have been skipped when it does not receive a direct fixation during the first pass through a sentence. While reading English texts, about one third of words are initially skipped (Rayner, 1998, 2009), whereas for Chinese texts, where characters are the basic orthographic units, up to $40-60 \%$ of individual Chinese characters are skipped during normal reading (Inhoff \& Liu, 1998; Liversedge et al., 2014). Word/character skipping can be influenced by two sources of information: (1) readers can preprocess words/characters that have not yet been fixated in the parafovea; and (2) readers can use the preceding sentence context to predict the upcoming words/characters. Past research has found that readers use both sources of information, resulting in a greater probability of skipping upcoming words and characters that are short, visually simple, and/or frequent, and thus easier to process parafoveally compared to those that are long, visually complex, or infrequent, as well as a greater probability for skipping words and characters that are predictable compared to those that are unpredictable (Rayner, 1998, 2009; Zang, Liversedge, Bai \& Yan, 2011, for reviews). However, it is still not clear how, and to what extent, these parafoveal and contextual sources information interact in determining whether a word or character is skipped or fixated. Are the effects of parafoveal processing and context additive, or is the effect of one dependent on the other? For example, context effects may only appear for words that are reasonably easy to process parafoveally. On the other hand, more parafoveal processing may take place when a word is also predictable compared to when it is not. 
The issue of word skipping is central to computational models of eye movement control during reading such as E-Z Reader (Reichle, 2011; Reichle \& Drieghe, 2013; Reichle, Rayner, \& Pollatsek, 2003; Reichle, Warren, \& McConnell, 2009) and SWIFT (Engbert \& Kliegl, 2011; Engbert, Nuthmann, Richter, \& Kliegl, 2005; Schad \& Engbert, 2012). Both models address the importance of parafoveal preprocessing and preceding sentence context in relation to word skipping, though there is a debate regarding the extent of the parafoveal processing that occurs prior to skipping. Specifically, E-Z Reader assumes that words are processed serially and sequentially, and the oculomotor system decides to intentionally skip a word when parafoveal processing of the upcoming word results in completion of the familiarity check (the first stage, $L_{1}$, of the identification of the word). This occurs with increased likelihood when the word is short, highly frequent or predictable from the context. In contrast, SWIFT assumes that multiple words within the perceptual span are processed simultaneously, and that word skipping can occur on the basis of incomplete parafoveal processing. Therefore, highly predictable words can be skipped if the identity of those words has been predicted from the preceding context without (or with minimal) visual input.

Recently, Angele and Rayner (2013) directly examined the relative importance of parafoveal processing and prior contextual information on skipping of the high frequency three-letter article the in reading. In the experiment, a gaze-contingent boundary paradigm (Rayner, 1975) was used, and participants were required to read each sentence containing a three-letter target verb (e.g., ace) with three parafoveal 
previews: a correct control preview (ace), an incorrect article preview (the), which was always syntactically illegal given the preceding context, or a nonword $(f d a)$. When the eyes crossed an invisible boundary that was located just to the left of the target position, the preview was replaced by the target verb, and thus readers always saw the correct verb. Angele and Rayner found that readers were more likely to skip the word in the target position (indeed, the skipping probability was nearly $50 \%$ ) when they had received the incorrect the previews compared to the other conditions, even though the article was syntactically inappropriate from preceding sentential context. This result clearly indicates that the decision to skip a word whose preview looks like the is mainly based on the parafoveal information available about the word. The constraints of the preceding sentence context do not seem to be taken into account. In a subsequent experiment, Angele, Laishley, Rayner and Liversedge (2014) extended this work using a similar paradigm but used three-letter content words of either high or low frequency as the syntactically illegal previews. They replicated the findings of Angele and Rayner (2013), and further demonstrated that readers made their skipping decision based on the parafoveal information only: high-frequency previews were frequently skipped even when they did not fit the sentence context. Further following up on this research, Abbot, Angele, Ahn, and Rayner (2015) investigated whether a the preview is preferentially skipped even if it occurs in a context that is highly constrained towards a different target verb, e.g. the as a preview for fit in the sentence "John found his old suit but it no longer fit properly at all."

Abbot et al. (2015) found that this was indeed the case. Most strikingly, the 
context effect seemed to be completely independent from the preview content, to the point that the previews were skipped more often when they occurred in a context that was predictable for the actual target verb than when they occurred in a context that was not predictable. Additionally, they successfully simulated the pattern of results using the E-Z Reader 10 model framework (Reichle et al., 2009), showing that a serial processing approach with a subsequent integration stage is, in principle, compatible with the notion of completely independent context and parafoveal processing effects.

Finally, in this special issue, Abbott and Angele (under submission) report that the tendency to skip high-frequency words preferentially despite incompatibility with the sentence context is not limited to three-letter words and therefore unlikely to be related to oculomotor saccade targeting issues (i.e. persistent overshooting of three-letter words). In summary, it is now quite well established that, in English, processing of the sentence context and parafoveal processing seem to operate independently in terms of making the decision to skip the upcoming word. Thus far, however, it is less than clear whether this might generalize to reading in a different language. One might speculate that most languages that employ articles (e.g. most Western European languages such as Spanish, French, Italian, German, Dutch, etc.) might show similar effects, as these languages share many features (such as the same alphabet). However, would we see similar effects in a completely different, non-alphabetic language that does not use articles?

In the present study we investigated whether contextual information can modulate the effect of parafoveal processing on word skipping in Chinese or whether 
contextual constraint and parafoveal processing operate completely independently with regards to word skipping like in English. Chinese is an ideal language to generalize this paradigm to, as it uses a very different writing system compared to the English language, namely a logographic writing system in which text is composed of characters which all occupy the same square unit of space. Characters are created from differing numbers of strokes and vary substantially in terms of visual and linguistic complexity. Compared to English, visual information in Chinese text is more densely packed, and therefore, more information may be visible to the right of fixation (Yan, Richter, Shu, \& Kliegl, 2009; Yang, Wang, Xu, \& Rayner, 2009; Zang et al., 2011). Thus Chinese readers may be able to process the parafoveal word to a greater degree than is the case in English before making a skipping decision. On the other hand, Chinese is an unspaced language without explicit visual markers to demarcate word boundaries (Bai, Yan, Liversedge, Zang, \& Rayner, 2008; Hoosain, 1992; Li, Zang, Liversedge, \& Pollatsek, 2015). As a consequence of this characteristic, the difficulty of segmenting words in the parafovea may lead to readers rely more heavily on the preceding sentence context to predict the identity of the upcoming words.

Chinese has no articles (i.e., no counterpart words to the), but the structural particle de (的) is an excellent candidate to examine Chinese word skipping, as it occurs extremely frequently and carries little meaning. The principal function of $d e$ is to link attributive modifiers with their head words or phrases (Lv, 2003; Yin, 1990). The attributive preceding de may be a noun (老师的书, lăo shì de shī meaning the 
teacher's book), pronoun (我的书, wǒ de shū meaning my book), verb (刚出版的书, gāng chū băn de shū meaning a newly published book), adjective (漂亮的书, piāo liàng de shū meaning a nice book), a numeral-measure word phrase (一桌的书, $y \bar{I}$ zhuō de shū meaning a table full of books), a prepositional phrase (关于心理学的书, guān yú xīn lǐ xué de shū meaning a book about psychology), as well as a number of other possibilities. The head word or phrase is always a noun or noun phrase. The relationship between attributive modifier and head word can be possessive, modifying, restrictive, etcetera. When the particle de is used to mark possession, it works like the possessive apostrophe $s$ in English. However, as mentioned above, in Chinese this word is used far more widely than is the possessive apostrophe s in English.

We employed the boundary paradigm and investigated to what extent Chinese readers use parafoveal information and prior sentence context information during skipping decisions. Participants read sentences containing a single-character target verb (e.g. 取, meaning get) whose preview was manipulated in three condition: identity; the high frequency structural particle $d e$ (which, in the position of the target character, was highly syntactically anomalous), or a pseudocharacter. If Chinese readers' decision to skip is based mainly on parafoveal information and is not modulated by the conflict between context information and parafoveal information, then they should skip the target at least as frequently for de previews as for identity previews. Alternatively, if Chinese readers, in contrast to English readers, can take sentence context into account when making skipping decisions based on parafoveal information, then they should detect the syntactic anomaly and be less likely to skip 
the target when the preview is the syntactically anomalous word de than when it is the identity preview. Finally, readers should skip the target less often and fixate it for longer when the preview is a pseudocharacter compared to an identity preview.

\section{Method}

\section{Participants}

Forty-five Tianjin Normal University students, with an average age of 22 (SD = 2) years, participated in the experiment. All participants were native Chinese speakers and had normal or corrected-to-normal vision. They were all naïve regarding the purpose of the experiment.

\section{Apparatus}

An SR Research EyeLink1000 eye tracker (sampling rate $=1000 \mathrm{~Hz}$ ) was used to record participants' eye movements. Participants read sentences presented on a 17-inch SAMSUNG SyncMaster 959NF monitor with a $1,024 \times 768$ pixel resolution and a refresh rate of $120 \mathrm{~Hz}$. The stimuli were presented in Song font in black on a white background. Viewing was binocular while only eye movements of the right eye were recorded. Viewing distance was $61 \mathrm{~cm}$, and each Chinese character subtended approximately $1.0^{\circ}$ of visual angle.

\section{Materials and design}

Sixty-nine single-character words were selected as target words from a database developed by Cai and Brysbaert (2010). All target words had left-right structure and were high frequency verbs, with a range from 53 to 931 occurrences per million $(M=$ $182, S D=186)$ and 5 to 11 strokes $(M=8, S D=2)$. Note that the structural particle $d e$ 
(的) had the highest frequency (50,155 per million) in the corpus and it had 8 strokes. Target words were embedded into sentences, which were all between 16 and 22 characters in length $(M=19, S D=2)$. All the sentences were rated for their naturalness and predictability. Forty-five participants (15 in each of the three counterbalancing conditions) who did not take part in the eye tracking study were asked to rate naturalness of sentences on a 5-point scale $(1=$ very unnatural, $5=$ very natural). The mean naturalness score for sentences containing the correct target verbs was $4.0(S D=0.3)$. For the predictability norms, another 18 participants performed a cloze task (i.e. guessed the following words given the sentence context up to the target words). The mean predictability score was very low $(M=0.6 \%, S D=1.9 \%)$, indicating that all target words were unpredictable from the prior sentence contexts.

The gaze-contingent boundary paradigm (Rayner, 1975) was used, in which a parafoveal preview stimulus was replaced by the target word when readers' eyes crossed the invisible boundary located before the target position. There were three preview conditions: the preview was either identical to the target word, a syntactically illegal particle word $d e$, or a pseudocharacter preview. The number of strokes was counterbalanced among the three preview conditions (it was always around 8 strokes given that $d e$ has 8 strokes), $t s>1.31, p s>.05$ (see Figure 1).

Insert Figure 1 about here

We constructed three files, with each file containing 69 sentences. There were 23 sentences in each condition, and conditions were rotated across files according to a Latin Square. Six practice sentences, two for each preview condition, were included at 
the beginning of each experimental file. In addition, there were 24 filler sentences without any changes that appeared throughout each file. $37 \%$ of sentences had Yes/No comprehension questions following them. Each participant read experimental and filler sentences presented randomly from one of the three files. In total each participant read 99 sentences.

\section{Procedure}

Participants were tested individually. They were informed that they would read sentences silently for comprehension, and would be presented with comprehension questions after the display of sentences occasionally. They gave answers to the comprehension questions by pressing a response key, and their answers were recorded by the computer. At the beginning of the experiment, there was a calibration procedure, after a successful calibration, participants read practice sentences to become familiar with the procedure. Then, the experimental sentences were presented in turn. The whole experiment lasted approximately 25 minutes.

\section{Results}

Participants' comprehension accuracy was 94\%, suggesting that they read and fully understood the sentences. Fixation durations shorter than $80 \mathrm{~ms}$ or longer than $1200 \mathrm{~ms}$ were excluded from the analyses. Trials were removed if (1) a track loss occurred or there were fewer than five fixations in total $(0.1 \%$ of the data); (2) eye movement measures were above or below three standard deviations from each participant's mean (for the target analyses: $0.6 \%$; for the pretarget analyses: $0.7 \%$; for the posttarget analyses: $0.8 \%$ ); (3) a blink occurred during display changes or during a 
fixation on the target word, as well as trials in which the display changes occurred untimely or delayed (11.9\%).

Skipping and fixation times on target, pretarget and posttarget characters were examined. Specifically, we calculated skipping probability (SP), first fixation duration (FFD, the duration of the first fixation on a character during first pass reading), single fixation duration (SFD, the fixation duration when only one fixation was made on a character during first pass reading), gaze duration (GD, the sum of all fixations on a character before moving to another character during first pass reading), go-past time (go-past, the sum of all the fixations from the first fixation on a character until the reader leaves the character to the right), and the probability of making regressions out (regressions from a region to a region earlier in the sentence). The means and standard deviations for the eye movement measures are shown in Table 1.

\section{Insert Table 1 about here}

To analyze the data, linear mixed models (LMM) were conducted using the lme4 package (version 1.1-7; Bates, Maechler, Bolker, \& Walker, 2015) in $\mathrm{R}(\mathrm{R}$ Development Core Team, 2014). For all measures on the target, pretarget and posttarget character, we ran the LMM with the maximum random effects structure (Barr, Levy, Scheepers, \& Tily, 2013), allowing both random intercepts and random slopes for the preview effect over both participants and items. However, if the "full" random model did not converge for a dependent measure due to missing values related to the high skipping rates, then we ran a model with intercepts and slopes for the preview effect with participants as a random factor and with intercepts for the 
items as random factors. Fixed effect estimations for the eye movement measures are shown in Table 2.

\section{Insert Table 2 about here}

\section{The Pretarget Character}

The de preview produced longer fixation times on, and more regressions from the pretarget character relative to the identity preview (Identical vs $d e$, FFD: $b=0.05$, $S E=0.02, t=1.88 ; \mathrm{SFD}: b=0.04, S E=0.03, t=1.67 ; \mathrm{GD}: b=0.05, S E=0.02, t=$ 2.05; Go-past time: $b=0.09, S E=0.03, t=3.12$; Regressions out: $b=0.62, S E=0.27$, $z=2.31$, though the effect was mariginally reliable for FFD and SFD), which constitutes a parafoveal-on-foveal effect (though note that adjacent characters were very close and they may have fallen within foveal vision in many cases, Inhoff, Radach, Starr, \& Greenberg, 2000; Zhou, Kliegl, \& Yan, 2013). The de and pseudocharacter conditions did not differ from each other across all these measures ( $p$ s $>.05$ ), suggesting that, for this particular parafoveal-on-foveal effect, it did not matter whether a real character or a meaningless character was in the parafovea; as long as it was unusual, it produced the effect. It is possible that, occasionally, readers process the content of the parafovea to a greater extent when they are planning to skip a parafoveal word, and if they do, this may increase their sensitivity to unusual parafoveal information. Alternatively, readers may skip a parafoveal word only if they happen to have processed it more deeply before making the skipping decision. However, post-hoc analyses using subsequent target skipping as an additional predictor showed no evidence of the preview effects on FFD, SFD, and GD being 
modulated by whether the target was subsequently skipped or not (all $|t|<1.96$ ). On go-past time, the difference between the identical and the de preview did seem to be reduced when the target word was subsequently skipped (Go-past time: $b=-0.20, S E$ $=0.06, t=-3.13)$. Based on this finding, it appears that the syntactic and parafoveal anomalies are occasionally detected very early. This is similar to the finding by Angele and Rayner (2013) and the subjsequent findings by Abbott et al. (2015) and Abbott and Angele (under submission) who showed that the pre-target word was skipped more often when the preview for the target word was the, even though the always appeared in a syntactically illegal context. No other effects were significant $(p \mathrm{~s}>.05)$

\section{The Target Character}

For skipping probability, readers skipped the target characters most often in the de preview (.58), less often in the identical preview (.51), and least in the pseudocharacter preview (.45) (Identical vs $d e: b=0.34, S E=0.13, z=2.64$; de vs pseudocharacter: $b=-0.62, S E=0.13, z=-4.63)$. Thus, Chinese readers were least likely to skip a parafoveal character when it was visually unfamiliar to them, as was the case in the pseudocharacter preview condition. More importantly, given our theoretical hypotheses, they skipped the target character more often in the semantically and syntactically anomalous de preview condition than in the identical preview in which the preview was compatible with the preceding context. And they skipped the target character more often in the identical preview condition than in the pseudocharacter preview condition. This indicates that the decision to skip de is 
strongly influenced by parafoveal information, and far less by a word's likelihood given sentential context.

For reading time measures where target characters were not skipped (note that, depending on the preview condition, this corresponds to only roughly $50 \%$ of the trials), readers spent longer fixating the target character after a pseudocharacter preview than after the de preview (de vs pseudocharacter, FFD: $b=0.08, S E=0.03, t$ $=3.01$; SFD: $b=0.12, S E=0.03, t=3.94$; GD: $b=0.11, S E=0.03, t=3.68$; Go-past time: $b=0.13, S E=0.04, t=3.11$ ). There was a numerical trend such that reading times on the target character following the de preview were longer, and the probability of making regressions out of the target character were higher, than those following identical previews, though the differences did not approach significance $(p>.05)$.

\section{The Posttarget Character}

For skipping probability, readers were less likely to skip the posttarget character in the pseudocharacter preview than in the de preview condition (de vs pseudocharacter: $b=-0.33, S E=0.10, z=-3.16$ ), there was no difference between the identical preview and the de preview conditions $(p>.05)$.

For reading time measures, there was no difference between the identical and de preview conditions for the early measures like FFD, SFD and GD ${ }^{1}$. However, go-past times following the de previews were reliably longer, and the probability of making a regression from the posttarget character was reliably greater than following the identical previews (Identical vs de, Go-past time: $b=0.23, S E=0.04, t=5.62$; Regressions out: $b=1.14, S E=0.18, z=6.37)$. These effects are consistent with a 
comparatively late disruptive effect of the de preview, presumably due to the difficulty associated with the integration of the posttarget character (and the target character, if it was not skipped) into the sentence structure, again, a finding that replicated the findings of Angele and Rayner (2013) and colleagues.

\section{Discussion}

The present study used a gaze-contingent boundary paradigm and manipulation similar to Angele and Rayner (2013), to provide Chinese readers with incorrect (highly frequent but syntactically anomalous) previews of the particle word de (的), to differentiate the role of parafoveal processing and the sentence context in Chinese word skipping during reading. We had two hypotheses: if Chinese readers base their skipping decisions on parafoveal processing, then when the preview suggests that the upcoming word is an extremely frequent particle word such as de, they should initially tend to skip the target word. However, since the particle de is inappropriate and can never be syntactically predicted on the basis of the preceding context, if readers make their skipping decision based on the preceding context, they should be less likely to skip the target word.

The present results are very straightforward and closely replicated the findings of Angele and Rayner (2013). There are four aspects of the results that are particularly noteworthy. First, we found clear evidence that Chinese readers were more likely to skip the target word when the preview was the high frequency particle de than when it was either an identity or a pseudocharacter preview, regardless of the fact that the identical preview was fully compatible with the preceding context but the preview de 
was not. This result suggests that readers made the decision to skip the parafoveal word based on its familiarity, and prior to fully identifying it and integrating its meaning into preceding context. That is to say, it appears that $d e$-skipping decisions were made based on the characteristics of the upcoming parafoveal character, rather than on its likelihood given the sentential context. At a broad level, these results demonstrate that parafoveal processing influences saccadic targeting in Chinese reading (see also Liu, Reichle \& Li, 2015; 2016). Furthermore, it seems likely that skipping decisions might be based on the visual familiarity of the parafoveal word or that, at the very least, parafoveal processing and contextual constraint have independent effects on skipping probability.

A second notable finding in the pre-target region was a parafoveal-on-foveal effect such that readers were more likely to regress when the preview was de than when it was the identity. In contrast to the skipping data at the target region, this effect does seem to point to readers being at least occasionally able to detect contextually anomalous parafoveal words (e.g., de). It could be argued that the skipping of the target region and regression data at the pretarget region are somewhat contradictory. If readers were aware that a parafoveal word was anomalous with respect to preceding context, why would they skip it? And, on the other hand, why would readers be more likely to skip a parafoveal word if they were sensitive to its contextual infelicity? In relation to these questions, it is perhaps useful to consider the mean values associated with the measures for the pre-target region. We can see that readers made a saccade from the pretarget word to skip it on approximately $50 \%$ of the trials (across 
conditions), and that they regressed from the pretarget word on $10 \%$ (identity) and $16 \%$ (de and pseudocharacter previews) of trials. We know that on the remaining trials readers must have moved their eyes to fixate the target (since these analyses for the pretarget character are based on the last fixation prior to a saccade from it). Given that readers regressed from the target word on between 15 and $23 \%$ of the occasions that they made a first pass fixation on it, then we can see that the proportion of trials for which we obtained evidence of a parafoveal-on-foveal effect is very small (approx. 10\% overall) relative to those for which we did not (approx. 90\% overall). In line with this logic, we suggest the following explanation for the pre-target region results. On the majority of fixations on the pre-target region, readers processed the parafoveal word to a relatively shallow level, and made a decision as to whether to skip it based on its visual familiarity. Thus, when the word was more familiar (e.g., de), skipping rates were higher than when the word was less familiar (e.g., identity), or less familiar still (e.g., pseudocharacter). On a small minority of trials, however, the parafoveal target word was processed to a level beyond visual familiarity, that is, at least to a point at which the parafoval word's infelicity with respect to preceding context was apparent. On this basis, readers may have detected the anomaly, experienced processing difficulty, and consequently regressed from the pretarget region in order to re-read the preceding context in an attempt to reconcile the anomalous word with that context. Thus, according to this explanation, and as we actually observed, regression rates should be increased for pseudocharacter and de previews relative to identity previews. Most notably, though, as the high skipping rate for the target word in the de preview 
condition showed, anomalies were either not detected often, or any such detection did not actually affect skipping decisions.

Third, the reading time data for the target, post target and a combined target and post target region fit neatly with this explanation. Recall that preview benefit on the target word was largest for identity preview, reduced for de previews and was smallest for pseudocharacter previews. (for the target character analyses the differences between the identity and de prevews did not approach significance, whereas for a combined target and post target region, the reading time measures showed reliable differences for GD and Go-past time). This is a standard finding in the literature both in Chinese reading (Zang et al., 2011 for a review) and reading in alphabetic languages (Rayner, 1975). Furthermore, these effects spilled over into the post target region such that the reading times were longest for pseudocharacter previews, shorter for de previews, and shortest for identity preview (again, though, in the post target character analyses, the differences for the early reading time measures, FFD, SFD and GD, were not significant between the identity and de preview conditions, and the de and pseudocharacter preview conditions). The spillover effects were comparable with previous findings in Chinese reading (e.g., Li, Bicknell, Liu, Wei \& Rayner, 2014) and alphabetic reading (e.g., Angele et al., 2014; Angele \& Rayner, 2013). However, it should be noted that there were longer go-past reading times and more regressions out of the post target region for the de previews compared to the identical previews, presumably reflecting difficulty associated with sentence integration processes (Angele \& Rayner, 2013). 
Finally, it should be noted that the present results are not contradictory in relation to previous findings regarding predictability and Chinese word skipping (e.g., Rayner, Li, Juhasz, \& Yan, 2005), which have shown that highly predictable words are more likely to be skipped than less predictable words. In contrast to the stimuli from this earlier work, all of our target words were unpredictable from the context, and this is likely why we did not see a pronounced effect of sentential context on Chinese word skipping. Both E-Z Reader and SWIFT models are able to account for these findings, at least with regard to skipping highly frequent particle de. E-Z Reader stipulates that the completion of the first stage of lexical acess, the familiarity check $\left(L_{1}\right)$ on word n, initiates a saccade program to move the eyes to word $n+1$. Specifically, if the eyes are on word $n$, and attention has shifted to word $n+1$ so that lexical processing of word $\mathrm{n}+1$ has begun, and the familiarity check on word $n+1$ in the parafovea is completed rapidly enough, then the eye guidance system will cancel the initial saccade to word $\mathrm{n}+1$ and instead make a saccade to fixate the following word $n+2$. As a consequence, word $n+1$ would be skipped. As the word de is very easy to process, it is likely that sufficient parafoveal processing could be carried out on that word to permit completion of the familiarity check before the saccadic program to initiate a saccade to this word has completed. Under such circumstances, readers will skip a de preview in the parafovea (indeed, analogously, the simulations performed by Abbott et al., 2015, indicate that the the skipping effect is compatible with E-Z Reader 10).

SWIFT assumes that words within an attentional gradient to the right of fixation will all be activated to differing degrees. Words within this gradient that are very easy 
to process will have activations that rise and then fall very rapidly (though these changes will themselves be modulated by the words' distances from fixation). The likelihood that the adjacent parafoveal word within the gradient will be skipped will be determined by that word's activation level relative to the activation level of other words in the gradient. When the the adjacent word's activation is high relative to the activation levels of the other words in the gradient, then it will likely receive a fixation. In contrast, when the activation level of the adjacent word is low relative to the activation level of words beyond it in the gradient, then it will likely be skipped. Thus, the likelihood that an adjacent parafoveal word will be skipped according to the SWIFT model, is dependent on how rapidly the activation level of that word rises to a maximum, and then declines rapidly relative to the concurrent levels of activation of other words within the attentional gradient. As the word de has the highest frequency in Chinese, its activation should rise and then decline most rapidly, and as a consequence it should not be a likely target candidate for the next saccade, and it should therefore be skipped. However a further question, then, is whether this effect is due to the word de being a particle or a high frequeny word, or both? And whether this effect is specific to the high frequency particle word de or it applies to other kinds of words that are highly frequent and less visually complex? This question requires further investigation.

To summarize, Chinese readers are likely to skip a high frequency parafoveal particle word de (的) without taking the syntactic sentence context into account, which replicates and extends the findings from English reading reported by Angele 
and Rayner (2013). The present study demonstrates whenever the word or character in the parafovea is highly visually familiar and easy to process, this may trigger an automatic skipping program overriding any source of constraint on reading behavior that derives from sentential context, and this phenomenon is observable across languages and writing systems that differ in their word spacing and visual density characteristics. 


\section{References}

Abbott, M.J., \& Angele, B (under submission). Preferential skipping of function words during reading. Manuscript submitted to QJEP Special Issue.

Abbott, M. J., Angele, B., Ahn, Y. D., \& Rayner, K. (2015). Skipping syntactically illegal the previews: the role of predictability. Journal of Experimental Psychology: Learning, Memory, and Cognition, 41(6), 1703-1714.

Angele, B., \& Rayner, K. (2013). Processing the in the parafovea: Are articles skipped automatically? Journal of Experimental Psychology: Learning, Memory, and Cognition, 39, 649-662.

Angele, B., Laishley, A.E., Rayner, K., \& Liversedge, S.P. (2014). The effect of highand low- frequency previews and sentential fit on word skipping during reading. Journal of Experimental Psychology: Learning, Memory, and Cognition, 40, 1181-1203.

Bai, X., Yan, G., Liversedge, S.P., Zang, C., \& Rayner, K. (2008). Reading spaced and unspaced Chinese text: Evidence from eye movements. Jornal of Experimental Psychology: Human Perception and Performance, 34, 1277-1287.

Barr, D., Levy, R., Scheepers, C., \& Tily, H.J. (2013). Random effects structure for confirmatory hypothesis testing: Keep it maximal. Journal of Memory and Language, 68, 255-278.

Bates,D., Maechler, M., Bolker,B., \& Walker, S. (2015). Fitting Linear Mixed-Effects Models using lme4. Journal of Statistical Software, 67(1), 1-48.

Cai, Q., \& Brysbaert, M. (2010). SUBTLEX-CH: Chinese word and character 
frequencies based on film subtitle. PLoS ONE, 5(6), e10729. DOI: 10.1371/journal.pone.0010729.

Engbert, R. \& Kliegl, R. (2011). Parallel graded attention models of reading. In Liversedge, S.P., Gilchrist, I.D. \& Everling, S. (Eds.), The Oxford Handbook of Eye Movements (pp.787-800). Oxford University Press.

Engbert, R., Nuthmann, A., Richter, E., \& Kliegl, R. (2005). SWIFT: A dynamical model of saccade generation during reading. Psychological Review, 112, 777-813.

Hoosain, R. (1992). Psychological Reality of the word in Chinese. In H.-C. Chen \& O. J.L. Tzeng. (Eds.), Language processing in Chinese (pp. 111-130). Amsterdam, Netherlands: North-Holland.

Inhoff, A. W., \& Liu, W. (1998). The perceptual span and oculomotor activity during the reading of Chinese sentences. Journal of Experimental Psychology: Human Perception and Performance, 24, 20-34.

Inhoff, A. W., Radach, R., Starr, M., \& Greenberg, S. (2000). Allocation of visuo-spatial attention and saccade programming during reading. In A. Kennedy, R. Radach, D. Heller, \& J. Pynte (Eds.), Reading as a perceptual process (pp. 221-246). Oxford, UK: North-Holland/Elsevier.

Li, X., Bicknell, K., Liu, P., Wei, W., \& Rayner, K. (2014). Reading is fundamentally similar across disparate writing systems: A systematic characterization of how words and characters influence eye movements in Chinese reading. Journal of Experimental Psychology: General, 143, 895-913. 
Li, X., Zang, C., Liversedge, S.P., \& Pollatsek, A. (2015). The role of words in Chinese reading. In A. Pollatsek \& Treiman, R. (Eds.), The Oxford Handbook of Reading (pp.232-244). Oxford University Press.

Liu, Y., Reichle, E.D., \& Li, X. (2015). Parafoveal processing affects outgoing saccade length during the reading of Chinese. Journal of Experimental Psychology: Learning, Memory, and Cognition, 41(4), 1229-1236.

Liu, Y., Reichle, E.D., \& Li, X. (2016). The effect of word frequency and parafoveal preview on saccade length during the reading of Chinese. Journal of Experimental Psychology: Human Perception and Performance, 42(7), 1008-1025.

Liversedge, S.P., Zang, C., Zhang, M., Bai, X., Yan, G, \&Drieghe, D. (2014). The effect of visual complexity and word frequency on eye movements during Chinese reading. Visual Cognition, 22, 441-457.

Lv, F. (2003). Comparative illustration of common Chinese words and expressions (pp. 142-144). Beijing, Beijing Language and Culture University Press.

R Core Team (2014). R: A language and environment for statistical computing. $\mathrm{R}$ Foundation for Statistical Computing, Vienna, Austria. URL http://www.R-project.org/.

Rayner, K. (1975). The perceptual span and peripheral cues in reading. Cognitive Psychology, 7, 65-81.

Rayner, K. (1998). Eye movements in reading and information processing: 20 years of research. Psychological Bulletin, 124, 372-422. 
Rayner, K. (2009). The thirty-fifth Sir Frederick Bartlett Lecture: Eye movements and attention in reading, scene perception, and visual search. Quarterly Journal of Experimental Psychology, 62, 1457-1506.

Rayner, K., Li, X., Juhasz, B.J., \& Yan, G. (2005). The effect of word predictability on the eye movements of Chinese readers. Psychonomic Bulletin \& Review, 12, 1089-1093.

Reichle, E. D., (2011). Serial-Attention Models of Reading. In Liversedge, S.P., Gilchrist, I.D. \& Everling, S. (Eds.), The Oxford Handbook of Eye Movements (pp.767-786). Oxford University Press.

Reichle, E.D., \& Drieghe, D. (2013). Using E-Z Reader to examine word skipping during reading. Journal of Experimental Psychology: Learning, Memory, and Cognition, 39 (4), 1311-1320.

Reichle, E.D., Rayner, K., \&Pollatsek, A. (2003). The E-Z Reader model of eye-movement control in reading: Comparisons to other models. Behavioural and Brain Sciences, 26, 445-476.

Reichle, E.D., Warren, T., \& McConnell, K. (2009). Using E-Z Reader to model the effects of higher level language processing on eye movements during reading. Psychonomic Bulletin \& Review, 16(1), 1-21.

Schad, D.J., \& Engbert, R. (2012). The zoom lens of attention: Simulating shuffled versus normal text reading using the SWIFT model. Visual Cognition, 20(4-5), $391-421$. 
Yan, M., Richter, E.M., Shu, H., \& Kliegl, R. (2009). Readers of Chinese extract semantic information from parafoveal words. Psychonomic Bulletin \& Review, $16,561-566$.

Yang, J., Wang, S., Xu, Y., \& Rayner, K. (2009). Do Chinese readers obtain preview benefit from word n+2? Evidence from eye movements. Journal of Experimental Psychology: Human Perception and Performance, 35, 1192-1204.

Yin, B. (1990). Chinese Romanization: Pronunciation and Orthography, Beijing, Sinolingua.

Zang, C., Liversedge, S.P., Bai, X., \& Yan, G. (2011). Eye movements during Chinese reading. In S.P. Liversedge, I. Gilchrist, \& S. Everling. (Eds.), The Oxford handbook of eye movements (pp. 961-978). Oxford University Press.

Zhou, W., Kliegl, R., \& Yan, M. (2013). A validation of parafoveal semantic information extraction in reading Chinese. Journal of Research in Reading, 36 (S1), S51-S63. 


\section{Acknowledgments}

We are grateful for support from the Recruitment Program of Global Experts (1000 Talents Award from Tianjin); Natural Science Foundation of China Grants $(31571122,81471629)$ and a scholarship from the China Scholarship Council. We are also grateful to Yanping Liu and two anonymous reviewers for their helpful comments on a previous version of the manuscript. 


\section{Footnote}

1. Due to the high initial skipping rate of a single Chinese character in the present study, there were no reliable first pass differences between the identical and the de preview conditions in the single target character analyses. However, by combining the target character and the post target character (identical across conditions), a region based analysis showed that FFD and SFD for the de previews were no longer than those for the identical previews. GDs and Go-past times were reliably longer for $d e$ than identical previews, and the probability of a regression out of the region was also reliably higher (GD: $b=0.09, S E=0.03, t=3.34$; Go-past time: $b=0.17, S E=0.04, t=4.27$; Regressions out: $b=0.39, S E=0.20, \mathrm{z}=1.94)$. These results were very comparable to the findings reported by Angele and Rayner (2013), which provide little evidence that the de preview had an effect on the earliest measures of target processing, but did affect the time readers spent re-reading the target and the text up to and including it. It seems likely that this was either caused by the increased target skipping during the first pass reading, or the difficulty of integrating $d e$ into an incompatible sentence context when $d e$ was identified before it was skipped. 